\title{
Effects of distributional information on categorization of prosodic contours
}

\author{
Chigusa Kurumada $^{1}$ (D) Meredith Brown $^{2,3}$ - Michael K. Tanenhaus ${ }^{1,4,5}$
}

Published online: 7 June 2017

(C) Psychonomic Society, Inc. 2017

\begin{abstract}
Although prosody clearly affects the interpretation of utterances, the mapping between prosodic representations and acoustic features is highly variable. Listeners may in part cope with this variability by adapting to distributions of acoustic features in the input. We examined whether listeners adapt to distributional changes using the construction It looks like an $X$. When pronounced with an $\mathrm{H}^{*}$ pitch accent on the final noun and a low boundary tone, the construction supports an affirmative interpretation (e.g., It looks like a ZEBRA [and I think it is one]). Conversely, when pronounced with a $\mathrm{L}+\mathrm{H}^{*}$ pitch accent and a rising boundary tone, it suggests a negative interpretation (e.g., It LOOKS like a zebra.... [but it is not]). Experiment 1 elicited pragmatic interpretations of resynthesized 12-step continua with these two contours as
\end{abstract}

Electronic supplementary material The online version of this article (doi:10.3758/s13423-017-1332-6) contains supplementary material, which is available to authorized users.

Chigusa Kurumada

ckuruma2@ur.rochester.edu

Meredith Brown

meredith@nmr.mgh.harvard.edu

Michael K. Tanenhaus

mtanenha@ur.rochester.edu

1 Department of Brain and Cognitive Sciences, University of Rochester, 304 Meliora Hall, Rochester, NY 14627, USA

2 Department of Psychology, Tufts University, Medford, MA, USA

3 Department of Psychiatry, Massachusetts General Hospital, Boston, MA, USA

4 Department of Linguistics, University of Rochester, Rochester, NY, USA

5 School of Psychology, Nanjing Normal University, Nanjing Shi, China the end points. In Experiment 2, one group of listeners heard items sampled from the most ambiguous region along the continua followed by affirmative continuations (e.g., It looks like a zebra because it has stripes all over its body) and items near the contrastive endpoint followed by negative continuations (e.g., It looks like a zebra but it is actually something else). Another group heard the reverse (i.e., ambiguous items with negative continuations and non-contrastive items with affirmative continuations). The two groups of participants subsequently derived diverging interpretations for novel ambiguous items, suggesting that prosodic processing involves flexible mappings between acoustic features and prosodic representations that are meaningful in interpretation of speech.

Keywords Prosody $\cdot$ Contrastive inference $\cdot$ Language comprehension $\cdot$ Adaptation

\section{Introduction}

Successfully conveying an idea depends not only on what a speaker says, but how he or she chooses to say it. Prosody, the melodic and rhythmic aspects of speech, undoubtedly plays an important role in conveying information about a speaker's beliefs and intentions that are not explicitly encoded in the content of an utterance (e.g., Büring, 2003; Cutler, 2015; Dahan, 2015; Gussenhoven, 2004; Jackendoff, 1972; Ladd, 2008; Pierrehumbert \& Hirschberg, 1990; Sag \& Liberman, 1975). However, how listeners map information from prosody onto pragmatic interpretations is not well understood.

In perhaps the most influential approach to mapping prosody onto speaker intentions, Pierrehumbert and Hirschberg (1990) proposed that listeners identify atomic units, such as pitch accents and boundary tones (Beckman \& Pierrehumbert, 1986; Liberman \& Pierrehumbert, 1984), which have unique 
functional meanings that combine to give rise to an overall intonational meaning. For instance, a high pitch accent aligned with a word stress (conventionally annotated as $\mathrm{H}^{*}$; Silverman et al., 1992) generally introduces new information into a discourse (e.g., I like APPLES $\mathrm{H}^{*}$ ) whereas an accent with low pitch followed by a steep pitch rise (i.e., L(ow)+ $\left.\mathrm{H}(\mathrm{igh})^{*}\right)$ highlights contextual contrast (e.g., I like APPLES_L+H*, but not pears).

While highly influential, this approach leaves open questions about how listeners recognize and process pragmatically meaningful intonational categories. The acoustic realization of intonational categories interacts with factors such as the stress pattern of an associated word, where in a sentence it appears, and more speaker- and/or situation-specific factors, such as overall pitch range, speech rate, and dialectal features (e.g., Arvaniti \& Garding, 2007; Ladd, 2008; Liberman \& Pierrehumbert, 1984). Therefore, mappings from acoustic cues to intonational categories are inherently non-deterministic, with values of acoustic cues overlapping across categories (Taylor, 2000). Even trained annotators often disagree on classification of intonational categories, which raises questions about how naïve listeners navigate variability in the input to arrive at coherent interpretations (e.g., Breen, Dilley, Kraemer, \& Gibson, 2012; Ladd \& Morton, 1997; Syrdal \& McGory, 2000).

The lack of invariance between the acoustic signal and more abstract linguistic categories is not specific to prosody. It has long been a central topic in spoken language processing, especially phonetic categorization (Liberman, Cooper, Shankweiler, \& Studdert-Kennedy, 1967). One way in which listeners cope with variability in mappings between acoustic features and phonemes is to expect variation and adapt to it by shifting expectations (e.g., Clayards, Tanenhaus, Aslin, \& Jacobs, 2008; Kleinschmidt \& Jaeger, 2015; Kraljic \& Samuel, 2007; Maye, Aslin, \& Tanenhaus, 2008; Norris, McQueen, \& Cutler, 2015). A powerful approach for examining adaptation in phonetic categorization is to use a continuum where the endpoints are clear tokens of phonemes that vary along a single dimension (e.g., VOT), determine a categorization function, and test whether this function is affected by exposure to different distributions of tokens. Extending this approach to adaptation with intonational contours presents challenges. First, unlike phonemes, pragmatically meaningful intonational categories are not easily accessible to naïve speakers (Dilley, 2010; Ladd \& Morton, 1997; Ladd, 2008). Second, different intonational categories can have overlapping interpretations (Watson, Tanenhaus, \& Gunlogson, 2008). Third, holistic impressions of pitch contours or emphases are based on multiple acoustic features (e.g., F0, duration, amplitude, intensity) that co-vary with one another and can span multiple phonemes, syllables, and words. Finally, interpretations of contours are heavily context dependent (e.g., Pierrehumbert \& Hirschberg, 1990).
Our solution uses a construction, It looks like an $X$, which can be associated with opposing interpretations depending on intonational contours. When used with a pitch accent on the final noun followed by a falling boundary tone (i.e., the $\mathrm{H}^{*} \mathrm{~L}$ L\% contour, or tune, which we refer to as the noun-focus contour; Fig. 1, left), this construction tends to evoke the affirmative interpretation [it looks like a zebra and it is one]. When instead used with a nuclear $\mathrm{L}+\mathrm{H}^{*}$ accent on the verb followed by a L-H\% boundary tone ("It LOOKS like a zebra...", which we refer to as the verb-focus contour; Fig. 1, right), it tends to trigger the negative interpretation [it looks like a zebra but it is not one] (Kurumada, Brown, Bibyk, Pontillo, \& Tanenhaus, 2014). In the verb-focus contour, the prosodically highlighted predicate looks like evokes the inference that the speaker intentionally chose looks like over the more frequent, and hence probable, predicate is to convey [It resembles a zebra but it is not] (see also Dennison \& Schafer, 2010).

To arrive at a particular pragmatic interpretation, listeners need to map the acoustic input onto increasingly abstract intonational and semantic representations (Fig. 2; for more discussion see Kurumada et al., under review). ${ }^{1}$ The current investigation postulates that intonational contours form partially overlapping distributions of intonational cues (e.g., pitch, duration, and intensity) that combine to guide listeners' interpretations. Due to the noise and variability associated with intonational contours, there are multiple intonational representations that can be induced from a given acoustic signal, as indicated with the dotted Gaussian distributions in Fig. 2. We assume, however, that listeners in our experiment have pragmatic expectations about the intended interpretation of It looks like an X (i.e. [it's an X] or [it's not an $\mathrm{X}]$ ) and would thus consider at least two contours corresponding to these interpretations (i.e. the noun- and verb-focus contours; Fig. 3).

We test the hypothesis that listeners adapt their assumptions about these distributions (e.g., means and variances) to accommodate variability in the input. In Experiment 1, we infer underlying distributional features of the intonational contours by eliciting categorization judgments for items sampled along a continuum interpolating $\mathrm{f} 0$ and duration between prototypical noun-focus and verb-focus contours. A steep categorization function would suggest that underlying distributions have smaller variances whereas a shallower function would indicate that at least one of the categories has relatively large variance (reflecting greater categorization uncertainty). In Experiment 2, we test the prediction that exposure to different

\footnotetext{
${ }^{1}$ For convenience we assume that we are measuring adaptation between acoustic cues and contours (tunes), which are composed of lower-level categories, such as pitch accents and boundary tones. We cannot rule out the possibility that the mapping also involves adaptation from acoustic cues to these phonological representations. This remains a question for future research.
} 


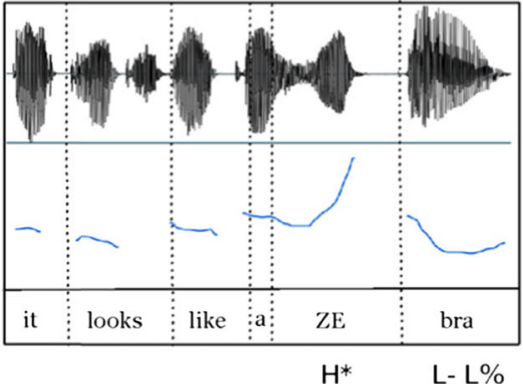

Fig. 1 Waveforms (top) and pitch contours (bottom) for the utterance It looks like a zebra, elicited from a native speaker of American English. The affirmative meaning [It is a zebra] is typically conveyed by the

distributions of items sampled from the continuum will result in adaptation of listeners' intonational categorization functions.

\section{Experiment 1: Determining the categorization function}

\section{Methods}

Participants Sixty participants used the online survey platform Amazon Mechanical Turk (https://www.mturk.com/). Participants were self-reported native speakers of American English, residing in the USA. They were advised to wear a headset or earphones. Mean task duration was $8 \mathrm{~min}$; participants received US $\$ 0.80$. We excluded four participants who completed the task in less than $100 \mathrm{~s}$ or more than $15 \mathrm{~min}$ or who made the same response to each item.

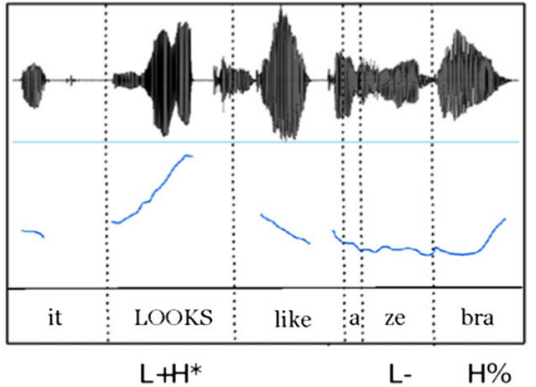

contour on the left (the noun-focus contour), while the negative meaning [It is not a zebra] is conveyed by the contour on the right (the verb-focus contour)

Stimuli A female speaker of American English (MB) recorded three tokens of It looks like an $X$ for 24 target items in each of the two target intonation contours. For the final noun, we chose 24 commonly known animal and object names, each disyllabic with initial stress (e.g., baseball, zebra). Six regions were identified and annotated within each recorded token, corresponding to the four initial words (i.e., it $\mid$ looks $\mid$ like $\mid$ a) and the portions of the final word associated with each of the two tonal targets: $\mathrm{H}^{*}$ and L-L\% in the noun-focus contour; L- and $\mathrm{H} \%$ in the verb-focus contour (Fig. 2). The peak or trough of the f0 contour within the final word (usually aligned with the first syllable) was used to delineate the final two regions. The $\mathrm{f} 0$ across each region was sampled at 20 equally-spaced time points. Measures from each time point were aggregated across items to derive mean $\mathrm{f} 0$ contours for noun-focus and verb-focus instructions, respectively, following Isaacs and Watson (2010). Mean durations for each region were similarly computed for both contours. We used the

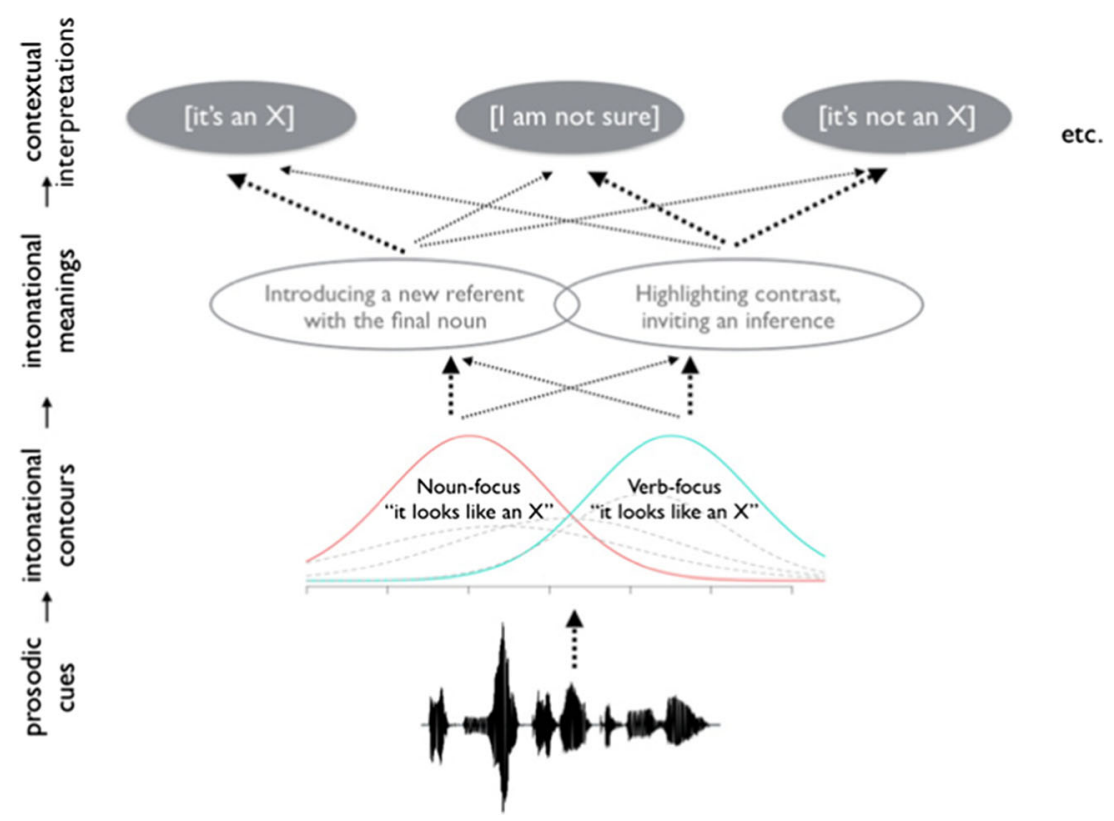

Fig. 2 Schematic illustration of multiple levels of mappings between the acoustic signal and higher level representations modified from Kurumada et al. (under review). Width of the arrows likelihoods of the mappings 

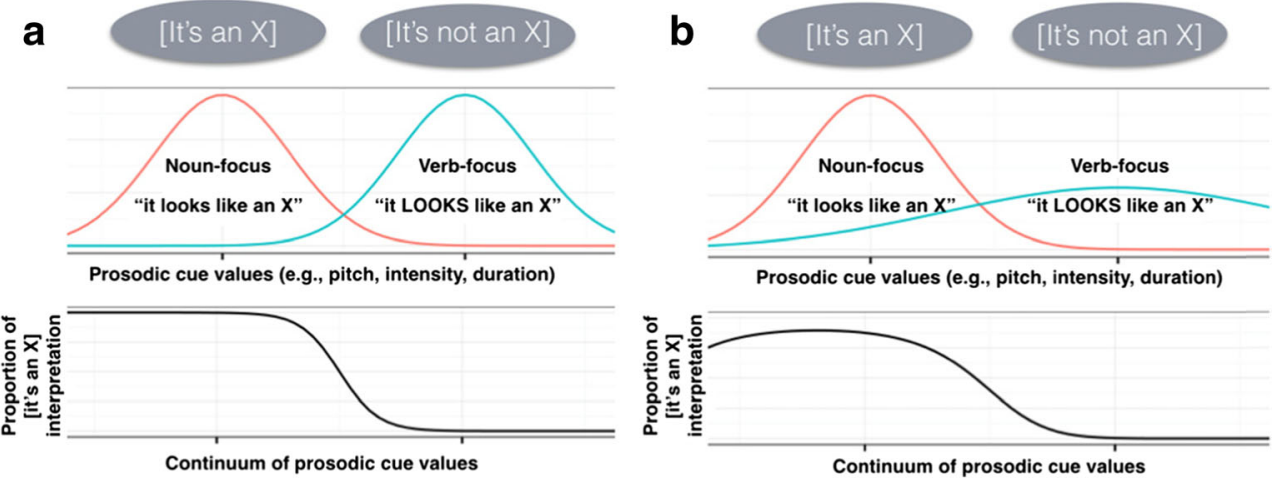

Fig. 3 Schematic illustration of distributional representations for the noun-focus and the verb-focus contours and categorization function curves for the distributions

pitch-synchronous overlap-and-add algorithm implemented in Praat (Boersma \& Weenink, 2008; Moulines \& Charpentier, 1990) to create a 12-step continuum for each item (Fig. 4). Acoustic cue values for each step were determined by interpolating ten equidistant steps between the mean f0 and duration values for the noun-focus and the verb-focus contours within each of the six regions. One recording of the verb-focus was then used as a base-form whose $\mathrm{f0}$ and duration values were replaced with the interpolated values. Sample sound files are provided in the Supplementary Material.

The endpoints of the continuum differ on multiple dimensions (e.g., nuclear accent location, segment duration, and combinatory patterns of pitch accent and boundary tone). We manipulated both pitch and duration to ensure that these co-varying dimensions shift together to maximize naturalness of the resynthesized stimuli. While this approach does not allow us to identify specific acoustic cues to which listeners adapt, demonstrating adaptation of intonation interpretations with these stimuli can serve as a springboard for more targeted questions about the nature of prosodic representations.

Procedure Participants were told that they would hear a mother's responses to her child's question, "What's on the next page?" Participants heard 24 tokens of It looks like an $X$ : two tokens per continuum step. Upon listening to each stimulus, participants were prompted to click on one of two candidate referents (Fig. 5): one prototypical target picture (e.g., a zebra) and one non-prototypical target picture (e.g., an okapi, a member of the giraffe family with zebra-like markings). Six lists were created by varying the nouns at each continuum step to mitigate possible spurious effects of itemspecific factors. For example, one list used zebra and table as Step 1 stimuli while another list used rabbit and candy as Step 1 stimuli. Order of presentation was randomized across participants. Participants did not receive feedback about which of the two referents was the intended target.

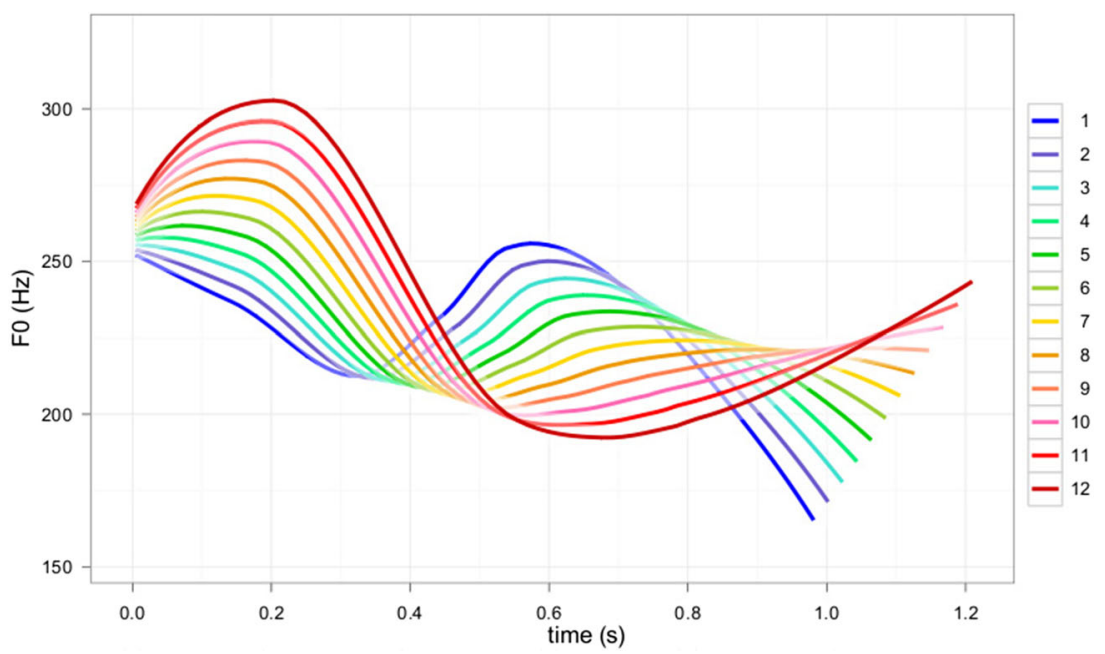

Fig. 4 Illustration of the resynthesized 12-step continuum. The bottom and top lines at time=0 are Step 1 (noun-focus) and Step 12 (verb-focus), respectively. The ten lines in between correspond to Steps 2-11 

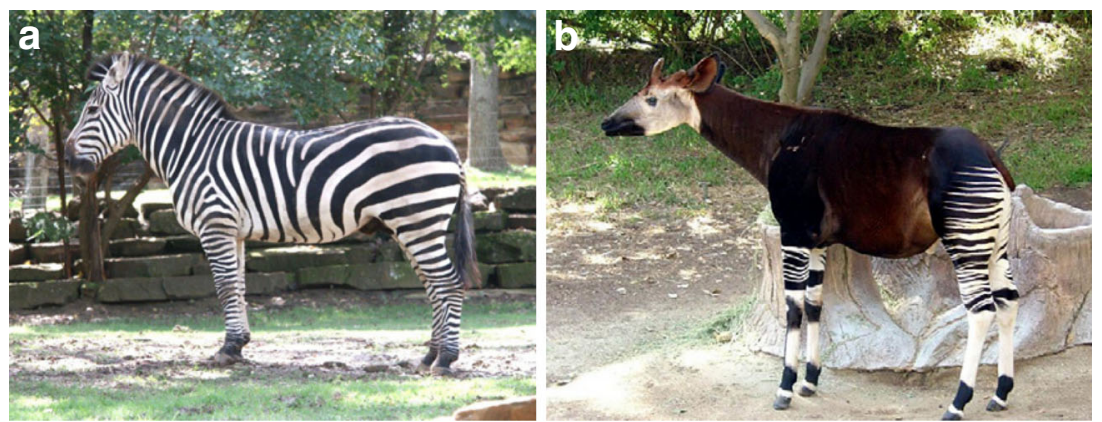

Fig. 5 Sample pictures presented in Experiment 1 for the target word zebra: (a) the prototypical target picture (a zebra), and (b) the non-prototypical target picture (an okapi). We expected (a) and (b) to be selected most frequently at Step 1 (noun-focus) and Step 12 (verb-focus), respectively

\section{Results}

Results are presented in Fig. 6. As expected, listeners were most likely to provide an affirmative (i.e., [It is an X]) interpretation for stimuli close to the prototypical noun-focus contour (i.e., Step 1). Judgments shifted toward [It is not an X] as stimuli became more similar to the prototypical verb-focus contour (i.e., Step 12). There was an overall bias toward the affirmative interpretation. Canonical noun-focus tokens at Step 1 elicited the affirmative interpretation on $84 \%$ of the trials compared to $37 \%$ for canonical verb-focus tokens at Step 12. The items for Step 10 were the most ambiguous (48\% affirmative responses).

The results are consistent with the hypothesis that listeners show gradient categorization judgments along the continuum (see Supplementary Material for additional analyses testing assumptions about the number of underlying distributions). The overall bias towards the affirmative response may reflect listeners' a priori bias towards recognizing a more generally frequent intonation contour (i.e., $\mathrm{H}^{*} \mathrm{~L}-\mathrm{L} \%$ ) and/or their tendency to derive a canonical, as opposed to pragmatically more complex, interpretation (see Supplementary Material for supporting information from past corpus

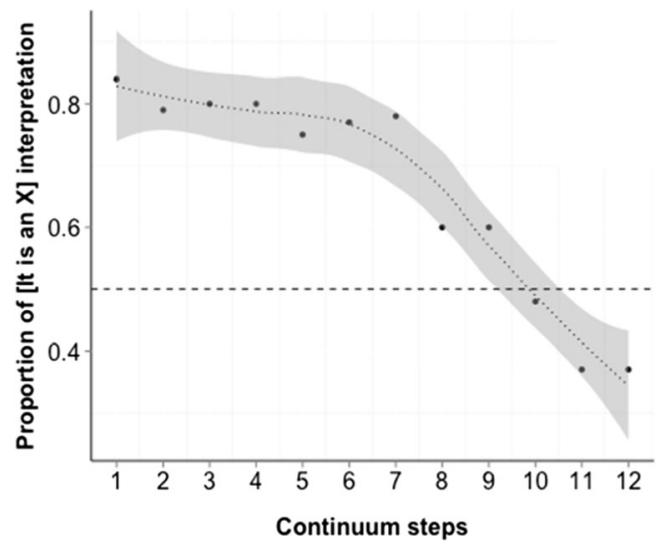

Fig. 6 Proportions of the affirmative [It is an X] interpretation by continuum step ( $1=$ noun-focus, $12=$ verb-focus). The dotted line represents loess smoothing, and shading indicates the $95 \%$ confidence interval studies). Consistent with previous observations in the literature, listeners' interpretation of prosody is thus systematic and yet exhibits considerable uncertainty. In Experiment 2, we examine whether listeners might navigate this uncertainty by adapting expectations for intonational contours.

\section{Experiment 2: Adaptation in interpretation of prosody}

\section{Methods}

Participants Three hundred and sixty participants completed the experiment using Amazon Mechanical Turk. We used the same exclusion criteria as Experiment 1 but extended the maximum task time to $25 \mathrm{~min}$ because of the increased number of trials. We obtained complete responses from 324 individuals.

Stimuli The 24 items from Experiment 1 were supplemented with five items recorded by the same speaker. Two items were used as example trials. Of the 27 remaining items, 15 items were used for the exposure phase, and 12 for the test phase. For each exposure item, two continuation phrases were recorded by the same speaker. One continuation supported the affirmative interpretation (e.g., [It looks like a zebra] "because it has black and white stripes all over its body"). The other supported the negative interpretation (e.g., [It LOOKS like a zebra] "but it's not. It has stripes only on its legs").

Procedure The experiment consisted of an exposure and a test phase. In the exposure phase, participants heard each exposure item twice: once with the affirmative continuation and once with the negative continuation (30 total trials). On each trial, they selected the prototypical or non-prototypical target picture as the more likely referent after hearing It looks like an X. After responding, they heard the continuation phrase 
accompanied by the corresponding prototypical or nonprototypical picture. Item order was randomized.

We created two exposure conditions (Fig. 7). In the no-shift condition, exposure items with affirmative continuations were sampled from Steps 4-9, and those with negative continuations from Steps 11-12 (Fig. 7a). In the negative-shift condition, exposure items with affirmative continuations were sampled from Steps 1-2, and those with negative continuations from Steps 7-12 (Fig. 7b). Consequently, items selected from Steps 7-9, which were judged to be relatively ambiguous in Experiment 1, differed in continuation phrase across conditions. In the no-shift condition, items from Steps 7-9 were disambiguated with affirmative continuations, thus approximating the distribution of mappings between acoustic features and meanings observed in Experiment 1. In the negative-shift condition, items from Steps 7-9 were instead disambiguated with negative continuations.

Each item in the exposure phase was preceded by one of two types of utterances from a second speaker (interlocutor utterances): "What's that?" or "It's an X!" (e.g., "It's a zebra!"). These interlocutor utterances were included to provide a plausible conversational context and thus make the endpoint judgments more symmetrical compared to Experiment 1. In particular, including "It's an X" interlocutor utterances makes the negative meaning of the verb-focus contour more readily available (e.g., Child: "It's a zebra!"; Mother: "It LOOKS like a zebra... [but it is not]"). Interlocutor utterances were recorded by a male native speaker of American English and manipulated with Praat's change gender command to make them sound like a child's voice. Eighty percent of the utterances with affirmative continuations were immediately preceded by a token of "What's that?" and 20\% were preceded by "It's an X!" to discourage listeners from relying solely on the preceding interlocutor utterances to guide their judgments. These proportions were reversed for utterances with negative continuations.

The test phase was identical across conditions. Participants made 2AFC judgments for 12 tokens of It looks like an $X$ (with no interlocutor utterance or continuation phrase) sampled from each of the 12 steps along the continuum. There was no overlap in target words between exposure and test phases. If categorization of intonation contours is modulated according to the distributional information in the input, exposure to the input in the negative-shift condition should result in recalibration of the categorization function, with more negative judgments compared to the no-shift condition.

\section{Results}

Responses are plotted in Fig. 8. As predicted, in the no-shift condition, participants' judgments were similar to those in Experiment 1, whereas in the negative-shift condition, a wider range of items elicited negative interpretations. Specifically, the proportion of affirmative interpretations dropped below $50 \%$ at Steps 7-9, which were associated with negative continuations during exposure. Likewise, items from Step 10, which were judged to be more or less ambiguous in Experiment 1 and in the no-shift condition, were more consistently assigned the negative interpretation.

We modeled judgments with multilevel logistic regression models implemented using the glmer function within the lme4 package in R (Bates, Mächler, Bolker, \& Walker, 2015; R Development Core Team, 2010). We included exposure condition (no-shift vs. negative-shift, contrast-coded), continuum steps (Steps 1-12, mean-centered, coded as a continuous variable), and their interaction as fixed effects. We used the maximum random effect structure justified by the data (Barr, Levy, Scheepers, \& Tily, 2013). When a model did not converge, we removed random slopes with the highest-order interaction terms accounting for the least variance. The final model contained random by-item intercepts and by-item slopes for exposure conditions.

We found main effects of condition $(\beta=.32, p<.001)$ and step $(\beta=-.18, p<.001)$. As predicted, participants in the negative-shift condition provided more negative responses. The interaction term was significant $(\beta=.05, p<.03)$. Participants in the negative-shift condition were more likely to provide negative responses for items closer to the higher end of the continuum than participants in the no-shift condition (i.e., Step 12). This suggests that listeners recalibrated

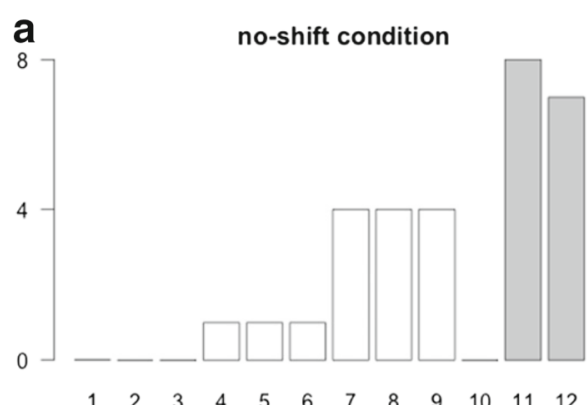

Fig. 7 Token counts of input sentences sampled from each step of the continuum (ranging from $1=$ noun-focus to $12=$ verb-focus) in the exposure phase of the (a) no-shift and (b) negative-shift conditions in

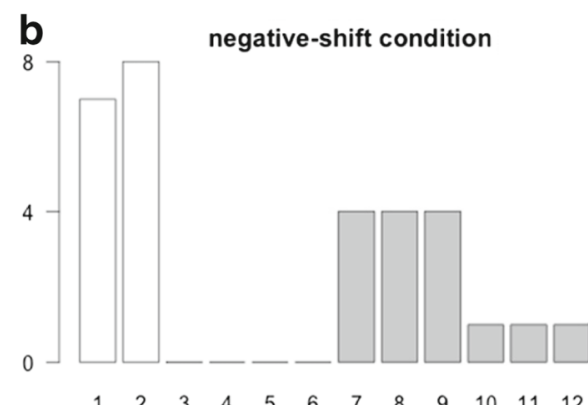

Experiment 2. White bars indicate tokens followed by affirmative continuations, and shaded bars those followed by negative continuations 


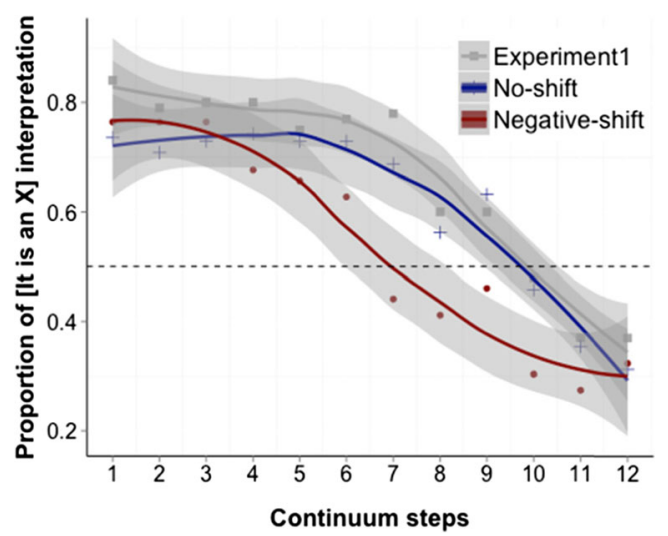

Fig. 8 Proportions of the affirmative [it is an $\mathrm{X}]$ interpretation in the test phase, by continuum step ( $1=$ noun-focus, $12=$ verb-focus). Gray, blue, and red lines represent responses from Experiment 1 (baseline) and the no-shift and negative-shift conditions from Experiment 2, respectively. Shaded areas indicate $95 \%$ confidence intervals

their interpretations of It looks like an $X$ to better reflect the statistics of the recent input from exposure items.

The current results cannot be solely attributable to pitch range normalization. If the exposure items were sampled from a narrower range in the negative-shift than in the no-shift condition, corresponding pitchrange, narrowing could lead listeners in the negativeshift condition to interpret subtle pitch excursion on LOOKS in the mid-range items as meaningful, boosting the negative interpretation for these otherwise ambiguous items. However, as illustrated in Fig. 7, the negative-shift condition included a wider range of intonation contours including extremes from the continuum (Steps 1-2 + Steps 10-12 in addition to the ambiguous middle range) than the no-shift condition (Steps 11-12 but not the other end of the continuum). We assume that the distinct response patterns between the conditions reflect a perceptual shift, which is optimized to map the variable acoustic input onto the two classes of pragmatically meaningful intonational contours. Without further investigations, however, we cannot rule out the possibility that the results represent a postperceptual shift in listeners' categorization judgments.

\section{General discussion}

We presented novel evidence that listeners' categorization of intonation contours can adapt in response to recent exposure. Past studies have reported variable mappings between the intonational input and its interpretations, but most focused on how different discourse contexts altered predictability of intonational contours and their likely interpretations (e.g., Büring, 2003; Calhoun, 2006; Gussenhoven, 2004; Jackendoff, 1972; Pierrehumbert \& Hirschberg, 1990). The current study demonstrated that distributional properties of the exposure items subsequently shifted interpretations of identical test items. After listening to only 30 exposure sentences, participants derived largely opposing responses to ambiguous intonational contours.

Crucially, categorization shifts in the negative-shift condition were induced by exposure to contours from only a subset of continuum steps. This shift would not be predicted if listeners were simply learning specific mappings between particular acoustic cue values and interpretations. Rather, the results suggest that listeners are extracting underlying distributional properties of phonological representations from which the observed examples were sampled. In future research it will be important to examine how listeners can dissociate corelated prosodic cue dimensions, such as those manipulated simultaneously in the current experiment, during adaptation (e.g., for a relevant discussion in phoneme perception see Liu \& Holt, 2015). It will also be important to examine to what extent speaker-specific information is stored as exemplars (Schweitzer, 2012) and whether adaptation generalizes across constructions and speakers.

Most generally the adaptation effects reported here provide a foundation for future work exploring how listeners store and represent prosodic information in order to efficiently and reliably process speech prosody despite variability across speakers and contexts.

\section{References}

Arvaniti, A., \& Garding, G. (2007). Dialectal variation in the rising accents of American English. Papers in Laboratory Phonology, 9, 547-576.

Barr, D. J., Levy, R., Scheepers, C., \& Tily, H. J. (2013). Random effects structure for confirmatory hypothesis testing: Keep it maximal. Journal of Memory and Language, 68(3), 255-278.

Bates, D., Mächler, M., Bolker, B., \& Walker, S. (2015). Fitting linear Mixed-Effects Models using lme4. Journal of Statistical Software 1(1).

Beckman, M. E., \& Pierrehumbert, J. B. (1986). Intonation structure in Japanese and English. Phonology Yearbook.

Boersma, P., \& Weenink, D. (2008). Praat: Doing phonetics by computer (Version 5.0.26) [Computer program].

Breen, M., Dilley, L. C., Kraemer, J., \& Gibson, E. (2012). Inter-transcriber reliability for two systems of prosodic annotation: ToBI (Tones and Break Indices) and RaP (Rhythm and Pitch). Corpus Linguistics and Linguistic Theory, 8(2).

Büring, D. (2003). On D-Trees, Beans, and B-Accents. Linguistics \& Philosophy, 26(5), 511-545.

Calhoun, S. (2006). Information structure and the prosodic structure of English: A probabilistic relationship. PhD Thesis. University of Edinburgh. 
Clayards, M., Tanenhaus, M. K., Aslin, R. N., \& Jacobs, R. A. (2008). Perception of speech reflects optimal use of probabilistic speech cues. Cognition, 108(3), 804-809.

Cutler, A. (2015). Native listening. MIT Press.

Dahan, D. (2015). Prosody and language comprehension. Wiley Interdisciplinary Reviews: Cognitive Science, 6(5), 441-452.

Dennison, H. Y., \& Schafer, A. J. (2010). Online construction of implicature through contrastive prosody. In Speech prosody 2010 conference.

Dilley, L. C. (2010). Pitch range variation in English tonal contrasts: Continuous or categorical? Phonetica, 67(1-2), 63-81.

Gussenhoven, C. (2004). The Phonology of Tone and Intonation. Cambridge University Press.

Isaacs, A., \& Watson, D. (2010). Accent detection is a slippery slope: Direction and rate of F0 change drives listeners' comprehension. Language and Cognitive Processes, 25(7), 1178-1200.

Jackendoff, R. (1972). Semantic interpretation in generative grammar. MIT Press.

Kleinschmidt, D. F., \& Jaeger, T. F. (2015). Robust speech perception: Recognize the familiar, generalize to the similar, and adapt to the novel. Psychological Review, 122(2), 148-203.

Kraljic, T., \& Samuel, A. G. (2007). Perceptual adjustments to multiple speakers. Journal of Memory and Language, 56(1), 1-15.

Kurumada, C., Brown, M., Bibyk, S., Pontillo, D. F., \& Tanenhaus, M. K. (2014). Is it or isn't it: Listeners make rapid use of prosody to infer speaker meanings. Cognition, 133(2), 335-342.

Ladd, D. R. (2008). Intonational phonology (2nd ed.). Cambridge University Press.

Ladd, D. R., \& Morton, R. (1997). The perception of intonational emphasis: continuous or categorical? Journal of Phonetics, 25(3), 313-342.

Liberman, A. M., Cooper, F. S., Shankweiler, D. P., \& Studdert-Kennedy, M. (1967). Perception of the speech code. Psychological Review, 74(6), 431-461.

Liberman, M., \& Pierrehumbert, J. (1984). Intonational invariance under changes in pitch range and length. In M. Aronoff \& R. Oehrle (Eds.), Language Sound Structure (pp. 157-233). Cambridge: MIT Press.
Liu, R., \& Holt, L. L. (2015). Dimension-based statistical learning of vowels. Journal of Experimental Psychology: Human Perception and Performance, 41(6), 1783-1798.

Maye, J., Aslin, R. N., \& Tanenhaus, M. K. (2008). The weckud wetch of the wast: Lexical adaptation to a novel accent. Cognitive Science, $32(3), 543-562$.

Moulines, E., \& Charpentier, F. (1990). Pitch-synchronous waveform processing techniques for text-to-speech synthesis using diphones. Speech Communication, 9(5-6), 453-467.

Norris, D., McQueen, J. M., \& Cutler, A. (2015). Prediction, Bayesian inference and feedback in speech recognition. Language, Cognition and Neuroscience, 3798, 1-15.

Pierrehumbert, J., \& Hirschberg, J. (1990). The meaning of intonational contours in the interpretation of discourse. In P. R. Cohen, J. Morgan, \& M. E. Pollack (Eds.), Intentions in communication (pp. 271-311).

R Development Core Team. (2010). Development Core Team, R: A language and environment for statistical computing. Vienna: $\mathrm{R}$ Foundation for Statistical Computing.

Sag, I. A., \& Liberman, M. (1975). The intonational disambiguation of indirect speech acts. Chicago Linguistic Society, 11, 487-497.

Schweitzer, K. (2012). Frequency effects on pitch accents: Towards an exemplar-theoretic approach to intonation. Ph.D dissertation, Universität Stuttgart.

Silverman, K., Beckman, M., Pitrelli, J., Ostendorf, M., Wightman, C., Price, P., Hirschberg, J. (1992). ToBI: A standard for labeling English prosody. In International Conference on Spoken Language Processing (Vol. 2, pp. 867-870). Banff.

Syrdal, A. K., \& McGory, J. (2000). Inter-transcriber reliability of ToBI prosodic labeling. Proceedings of the ICSLP-20003, 3, 235-238.

Taylor, P. (2000). Analysis and synthesis of intonation using the Tilt model. The Journal of the Acoustical Society of America, 107(3), 1697-1714.

Watson, D. G., Tanenhaus, M. K., \& Gunlogson, C. A. (2008). Interpreting pitch accents in online comprehension: $\mathrm{H}^{*}$ vs. $\mathrm{L}+\mathrm{H}^{*}$. Cognitive Science, 32(7), 1232-1244. 